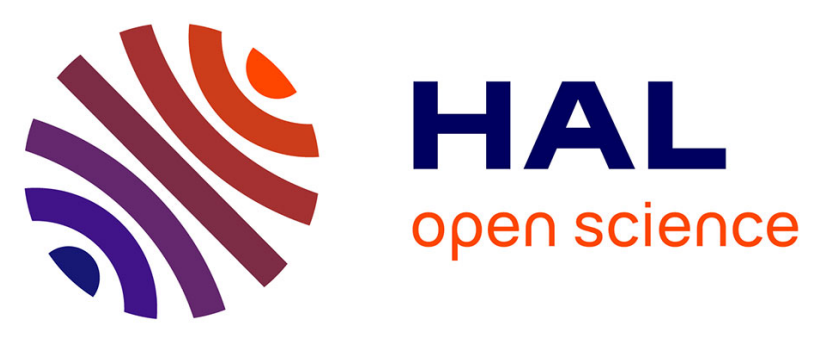

\title{
Decrease in thermal conductivity in polymeric P3HT nanowires by size-reduction induced by crystal orientation: new approaches towards thermal transport engineering of organic materials
}

\author{
Miguel Muñoz Rojo, Jaime Mart'In, Stéphane Grauby, Theodorian \\ Borca-Tasciuc, Stefan Dilhaire, Marisol Martin-Gonzalez
}

\section{To cite this version:}

Miguel Muñoz Rojo, Jaime Mart'In, Stéphane Grauby, Theodorian Borca-Tasciuc, Stefan Dilhaire, et al.. Decrease in thermal conductivity in polymeric P3HT nanowires by size-reduction induced by crystal orientation: new approaches towards thermal transport engineering of organic materials. Nanoscale, 2014, 6 (14), pp.7858-7865. 10.1039/C4NR00107A . hal-01025464

\author{
HAL Id: hal-01025464 \\ https://hal.science/hal-01025464
}

Submitted on 19 Feb 2016

HAL is a multi-disciplinary open access archive for the deposit and dissemination of scientific research documents, whether they are published or not. The documents may come from teaching and research institutions in France or abroad, or from public or private research centers.
L'archive ouverte pluridisciplinaire HAL, est destinée au dépôt et à la diffusion de documents scientifiques de niveau recherche, publiés ou non, émanant des établissements d'enseignement et de recherche français ou étrangers, des laboratoires publics ou privés.

\section{다(1)(2)}

Distributed under a Creative Commons Attribution - ShareAlikel 4.0 International 


\title{
Decrease in thermal conductivity in polymeric P3HT nanowires by size-reduction induced by crystal orientation: new approaches towards thermal transport engineering of organic materials $\uparrow$
}

\author{
Miguel Muñoz Rojo, ${ }^{a}$ Jaime Martín, ${ }^{a}$ Stéphane Grauby, ${ }^{b}$ Theodorian Borca Tasciuc, ${ }^{c}$ \\ Stefan Dilhaire ${ }^{\mathrm{b}}$ and Marisol Martin Gonzalez ${ }^{* a}$
}

To date, there is no experimental characterization of thermal conductivity of semiconductor polymeric individual nanowires embedded in a matrix. This work reports on scanning thermal microscopy measurements in a $3 \omega$ configuration to determine how the thermal conductivity of individual nanowires made of a model conjugated polymer (P3HT) is modified when decreasing their diameters. We observe a reduction of thermal conductivity, from $\lambda_{\mathrm{NW}}$ $2.29 \pm 0.15 \mathrm{~W} \mathrm{~K}^{1} \mathrm{~m}^{1}$ to $\lambda_{\mathrm{NW}} \quad 0.5 \pm 0.24 \mathrm{~W} \mathrm{~K}^{1} \mathrm{~m}^{1}$, when the diameter of nanowires is reduced from $350 \mathrm{~nm}$ to $120 \mathrm{~nm}$, which correlates with the polymer crystal orientation measured by WAXS. Through this work, the foundations for future polymer thermal transport engineering are presented.

Nanostructuring is used to modify and control the transport properties of materials due to confinement effects. For example, thermal conductivity reduction by size effects has yielded more efficient thermoelectric devices., ${ }^{\mathbf{1} 2}$ Among transport properties of materials, especially challenging are measurements of thermal conductivity, which become even more difficult as the dimensions of the material are reduced. ${ }^{3}$ However, the analysis of this physical property under nanoscale confinement is mandatory for a wide variety of technological applications ranging from thermoelectrics to nanoscopic thermal insulation, among others.

Generally, size effects on the thermal transport properties are dramatic for 1D nanostructures due mainly to scattering processes, as heat propagation is confined to a single spatial dimension. This has been theoretically predicted and experimentally observed for inorganic nanowires (NWs). ${ }^{3,4}$ However, little is known about how low dimensionality affects the thermal transport properties in semiconducting polymer

anstituto de Microelectrónica de Madrid, Calle de Isaac Newton, 828760 Tres Cantos, Madrid, Spain.E mail: marisol@imm.cnm.csic.es

${ }^{b}$ Univ. Bordeaux, LOMA, UMR 5798, 33405 Talence, France

${ }^{c}$ Rensselaer Polytechnique Institute, 110 8th St, Troy, NY 12180, USA materials, although severe changes are also expected, because nanoconfinement is well known to induce structural and dynamical changes in nanoconfined polymers. ${ }^{5}$ Thereby, our aim is to clarify how the thermal conductivity of relevant semiconducting polymer nanowires inside a matrix is altered by nanoconfinement.

Shen et $a .^{6}$ measured for the first time the thermal conductivity of single ultra-drawn polyethylene (PE) NWs and observed a dramatic increase of the thermal conductivity of the NWs when reducing diameters, which was correlated with the molecular orientation and reduction of voids and defects. Likewise, Cao et $a .^{7}{ }^{7}$ reported the enhancement of thermal conductivity of PE NWs. However, the measured NWs consisted of collapsed bundles of NWs and, thus, these measurements might be influenced by the different environments experienced by the NWs - NWs at interior positions of the bunch, from those at external positions, free NWs, etc. Therefore, to understand the thermal behavior of NWs it is mandatory to study the thermal transport properties of isolated NWs under well controlled boundary conditions. For our study, we have selected poly(3-hexylthiophene) (P3HT) as the model semiconducting polymer, as P3HT is one of the best characterized semiconducting polymers from a structural point of view. ${ }^{8}$ Furthermore, it has recently shown promising thermoelectric figures of merit at room temperature, for which the characterization of the thermal conductivity was crucial to calculate its efficiency. ${ }^{9}$ Although little is known regarding the confinement effects on thermal properties of P3HT, the few studies reported on P3HT $2 \mathrm{D}$ thin films have shown anisotropy of the thermal conductivity along different spatial dimensions. ${ }^{\mathbf{1 0 , 1 1}}$ However, measurements of individual NWs are extremely challenging due to the high spatial resolution required and only very few techniques are able to do it with accuracy. ${ }^{3}$

In this work, a technique called $3 \omega$-SThM (Scanning Thermal Microscopy) based on Scanning Probe Microscopy (SPM) ${ }^{12}$ was used to carry out the first local measurements of thermal conductivity on individual semiconducting polymer NWs. These measurements fill a gap in the literature and constitute a 
step toward the determination of how polymer materials behave at this low scale.

On the one hand, the most typical technique that is able to measure the thermal conductivity of single NWs is the microfabricated suspended device technique. ${ }^{13}$ Nevertheless, it is worth mentioning that this method, which measures only one NW at a time, requires many heavy processing steps and may lead to the oxidation of the surface of the NWs, since they are not embedded in a matrix but are in contact with air. In addition, this technique measures the thermal conductivity of one NW isolated from its matrix which can differ from the thermal conductivity of the NWs embedded in the matrix, which constitutes the effective functioning device, because of the matrix/NW interactions. ${ }^{\mathbf{1 4}}$ On the other hand, there are several techniques that can carry out local thermal characterization of arrays of nanowires embedded in a matrix, such as the time domain thermoreflectance (TDTR) ${ }^{14}$ the photoacoustic technique ${ }^{15}$ the photo-thermoelectric technique ${ }^{\mathbf{1 6}}$ or others based on micro-probe measurements. ${ }^{17}$ Nevertheless, the typical spatial resolution reached by these techniques is about $1 \mu \mathrm{m}$, which does not enable them to carry out thermal measurements on individual nanowires but gives access to a mean value of the thermal conductivity of the whole sample. $3 \omega$-SThM is a local technique that enables to capture thermal images of individual NWs with a $100 \mathrm{~nm}$ typical thermal spatial resolution and a 10 $\mathrm{nm}$ typical topographical spatial resolution. This technique has the advantage to probe thermally a wide range of individual NWs embedded in their matrix in short periods of time, ranging from 10 to $20 \mathrm{~min}$ depending on the signal generator frequency used. ${ }^{18}{ }^{20}$ We obtain at the same time a topographical image and a thermal image, enabling easy localization of the NWs, determining at the same time if the pore is filled, what is the morphological quality of the nanowires studied, etc. In addition from a single thermal image, we can assess as many NW measurements as the number of NWs in the image, leading to a mean value and a standard deviation of the measured signal among nanowires. ${ }^{21}$

Thermal conductivity measurements are carried out using $3 \omega$-SThM working in contact mode (see ESI, S2 $\dagger$ ). This technique has been recently used to measure the thermal conductivity of inorganic NWs of $\mathrm{Si}^{19}{ }^{19} \mathrm{SiGe}^{21}$ and $\mathrm{Bi}_{2} \mathrm{Te}_{3} \cdot{ }^{20}$ Classically, $3 \omega$-SThM measurements are performed using a Wollaston probe. ${ }^{22}$ However, Wollaston probes face two main drawbacks: a thermal spatial resolution around $1 \mu \mathrm{m}$ comparable to TDTR, which makes them unsuitable to probe individual NW measurements at nanometric scale, and a low thermal cut-off frequency which infers a low excitation frequency and hence a high acquisition time. $\mathrm{Pd} / \mathrm{SiO}_{2}$ probes used in our measurements present a $100 \mathrm{~nm}$ thermal spatial resolution and a cut-off frequency ten times higher than the Wollaston one. ${ }^{18}$ Thereby, in this work the thermal conductivity of individual P3HT NW (with diameters of $120 \mathrm{~nm}, 220 \mathrm{~nm}$ and $350 \mathrm{~nm}$ ) has been assessed by scanning thermal microscopy working in a $3 \omega$ configuration while being embedded in an alumina template.

The hexagonally ordered AAO templates with pores of 120 , 220 , and $350 \mathrm{~nm}$ diameter and $100 \mu \mathrm{m}$ length were synthesized by two-step electrochemical anodization of aluminum and subsequent chemical etching as reported in the literature for templates with pore diameter in the 120-400 nm range (23,24 $^{2}$ ESI, Fig. S1 $\dagger$ ). For the fabrication of P3HT NWs, macroscopic pieces of commercial P3HT from Aldrich Ltd. $\left(M_{\mathrm{n}}=33405 \mathrm{~g} \mathrm{~mol}^{-1}\right.$, $M_{\mathrm{w}} / M_{\mathrm{n}}=1.50$, region regularity $=96 \%$ ) were placed onto the surface of the AAO at $260{ }^{\circ} \mathrm{C}$ for $45 \mathrm{~min}$ in a $\mathrm{N}_{2}$ atmosphere. ${ }^{25}$ Then the samples were taken out from the furnace, and quenched in ice-water, so that P3HT rapidly solidifies. The excess of P3HT at the AAO top surfaces was removed with a razor blade and the surface was polished with diamond paste ( $3 \mu \mathrm{m}$, Buehler MetaDi II). SEM micrographs of the surface of the infiltrated templates are shown in the ESI, Fig. S1a and S1b. $\dagger$ Finally, the P3HT-infiltrated templates were annealed at $125{ }^{\circ} \mathrm{C}$ over $30 \mathrm{~min}$. A sketch of the samples is included in the ESI, Fig. S1c. $\dagger$

Wide-angle X-ray scattering (WAXS) experiments in a geometry in which the wave vector, $Q$, was parallel to the long axis of P3HT NWs were carried out in reflection geometry using a Philips X'Pert diffractometer (ESI, Fig. S3a †). Moreover, WAXS experiments were also carried out in transmission geometry with the X-ray beam traveling along the direction perpendicular to the template surface using a Bruker AXS Nanostar X-ray scattering instrument (see ESI, Fig. S3b $\dagger$ ), so that $Q$ was nearly perpendicular to the long axis of NWs. The underlying Al substrate was chemically etched from the AAO templates for transmission measurements. The scattered X-rays were detected using a two dimensional multiwire area detector (Bruker HiStar). The data were then converted to one-dimensional scattering profiles by radial averaging along the azimuthal direction. The sample to detector distance was $10 \mathrm{~cm}$. Both instruments use $\mathrm{Cu} \mathrm{K} \alpha$ radiation $(1.54 \AA$ ).

$3 \omega$-SThM was applied to measure P3HT NWs with different diameters embedded in a porous alumina matrix. It is worth mentioning that this experimental technique not only allows measurements of the thermal resistance, $R_{\mathrm{eq}}$, of individual NWs inside the matrix, but also gives information of the $R_{\text {eq }}$ of the whole composite. ${ }^{20}$ This technique is based on statistical data processing to determine the mean average of the equivalent thermal resistance of the NWs and the whole composite, with its associated standard deviation.

Fig. 1a shows Scanning Electron Microscopy (SEM) pictures of a top view of the un-filled porous alumina templates used to embed P3HT NWs, as well as topographic (Fig. 1b) and $3 \omega$ voltage $\left(\left(V_{3 \omega}\right)_{\text {Tip }}\right)$ (Fig. 1c) images of P3HT NWs with three different nanowire diameter sizes, $350 \mathrm{~nm}, 220 \mathrm{~nm}$ and $120 \mathrm{~nm}$, respectively. Additional SEM images of the P3HT NWs embedded in the template are shown in the ESI, Fig. S1a and S1b. $\dagger$

According to the $\left(V_{3 \omega}\right)_{\text {Tip }}$ thermal images of P3HT NWs, we can distinguish two areas in each of them: a high $V_{3 \omega}$ signal area corresponding with the NW locations and a low $V_{3 \omega}$ signal area on the alumina. Then, the NW mean equivalent thermal resistances $\left(R_{\mathrm{eq}}\right)_{\mathrm{NW}}$ for the three different diameters can be determined from the $V_{3 \omega}$ value measured on each NW. The results are shown in Table 1 . Let us underline that each $\left(R_{\text {eq }}\right)_{\text {NW }}$ value presented in this table was obtained after measurements on 20 NWs (even for the $120 \mathrm{~nm}$ NW sample for which we have used a 


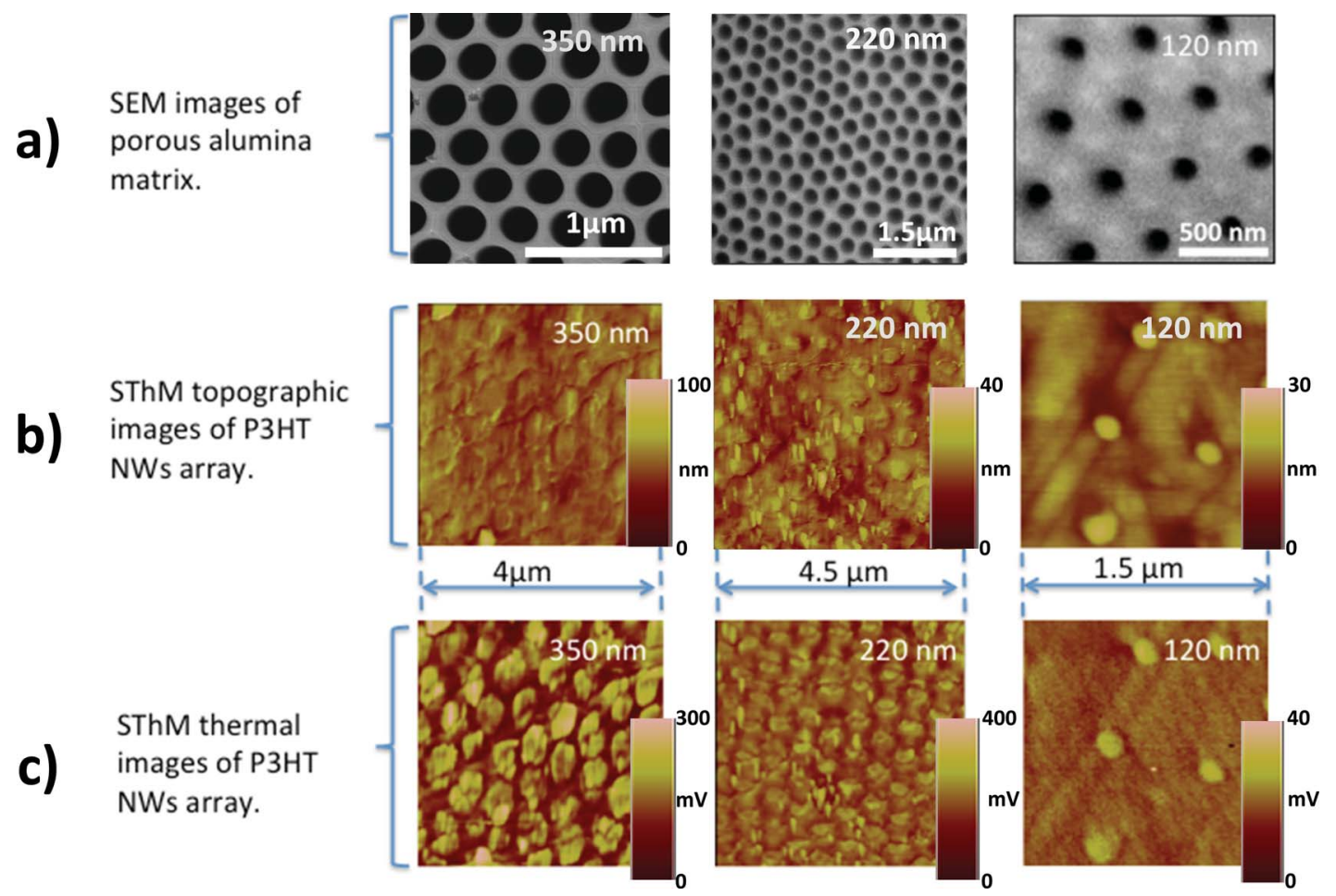

Fig. 1 (a) SEM pictures of the three different diameter size porous alumina matrices used to embed P3HT NWs, (b) topography of the filled templates and (c) $\left(V_{3 \omega}\right)_{T i p}$ or thermal images of P3HT NWs taken using $3 \omega-$ SThM.

thermal image larger than the one in Fig. 1c). In this table the thermal exchange radius of the tip $r_{\mathrm{ex}}$ is also included, whose value was specifically measured before each sample scan. It constitutes an important parameter to take into account when doing this analysis (ESI, S2 $\dagger$ ). Indeed, not only does it influence the spatial resolution but it is also a key parameter in the estimation of the thermal conductivity through the evaluation of some of the thermal resistances involved in the total equivalent thermal resistance measured, as developed below. ${ }^{18}$

After the evaluation of the equivalent thermal resistance $\left(R_{\mathrm{eq}}\right)_{\mathrm{NW}}$ on the NWs, one can determine the thermal conductivity of the NWs. For this purpose, one must consider that the equivalent thermal resistance measured can generally be expressed as the addition of 4 thermal resistances in series, the tip to sample contact thermal resistance $R_{\mathrm{C}}$, the constriction resistance $R_{\text {Tip-NW }}$ of the heat flux between the tip and the NW, the sample intrinsic thermal resistance $R_{\text {Com }}$, and the constriction resistance $R_{\mathrm{NW}-\mathrm{Sub}}$ of the heat flux between the NW and the substrate on which the composite is deposited. ${ }^{20}$ This is expressed by eqn (1),

$$
\left(R_{\mathrm{eq}}\right)_{\mathrm{NW}}=R_{\mathrm{Tip} \mathrm{NW}}+R_{\mathrm{C}}+R_{\mathrm{Com}}+R_{\mathrm{NW} \mathrm{Sub}}
$$

On the one hand, the constriction resistance between the tip and the NW, $R_{\mathrm{Tip}-\mathrm{NW}}$, is negligible as the thermal exchange surface is larger than the NW section, whose diameters vary from $350 \mathrm{~nm}$ to $120 \mathrm{~nm}$. On the other hand, the heat flows through the whole composite and, given that the matrix is 100 $\mu \mathrm{m}$ thick, the majority of the heat will not reach the substrate. Therefore, the constriction resistance between the NW and the substrate can be neglected. Therefore, eqn (1) is reduced to

$$
\left(R_{\mathrm{eq}}\right)_{\mathrm{NW}}=R_{\mathrm{C}}+R_{\mathrm{Com}}
$$

In order to determine $R_{\text {Com }}$ and subsequently $\lambda_{\text {Com }}$, it is now necessary to evaluate the thermal contact resistance, $R_{\mathrm{C}}$. As developed by Lefèvre $e t$ al., ${ }^{22}$ this resistance takes into account

Table 1 Areal packing density of the NW array and alumina, thermal exchange radius, equivalent thermal resistance and thermal conductivities of the composite, alumina matrix and intrinsic NWs for three different composites made of the P3HT NW array embedded in the alumina matrix

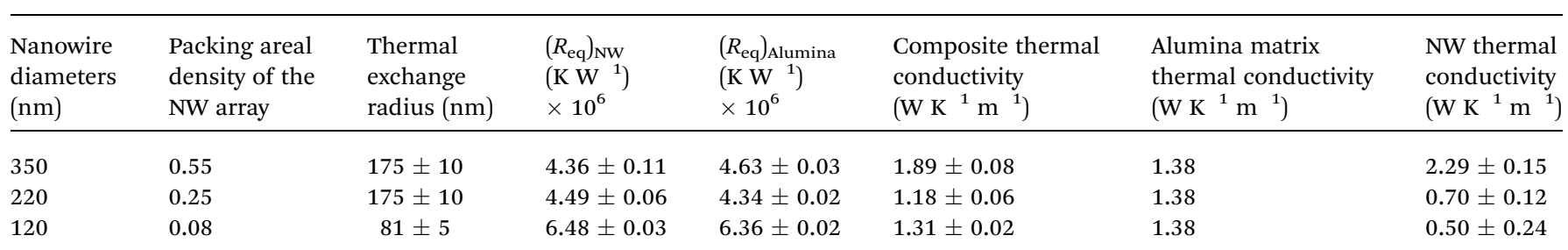


not only the solid-solid conduction between tip and sample, but also conduction through air and through the water meniscus, which constitutes the two other main heat transfer mechanisms under atmospheric conditions. It can be expressed as: $1 / R_{\mathrm{C}}=G_{\mathrm{C}}=G_{\mathrm{S}}+G_{\mathrm{A}}+G_{\mathrm{W}}$, where $G_{\mathrm{S}}, G_{\mathrm{A}}$ and $G_{\mathrm{W}}$ are respectively the conductance through the solid-solid contact, through air and through the water meniscus. The heat transfer mechanisms take place over a surface not defined by the contact-contact radius but by the thermal exchange radius $r_{\mathrm{ex}}$, and hence the necessity to calibrate this parameter. ${ }^{18}$

To evaluate $R_{\mathrm{C}}$ we measure the equivalent thermal resistance on the alumina matrix..$^{19,20,26,27}$ Indeed, in this case, the equivalent thermal resistance measured on the alumina is given by

$$
\left(R_{\mathrm{eq}}\right)_{\mathrm{Alu}}=R_{\mathrm{C}}+R_{\mathrm{Tip} \text { Alu }}
$$

where $R_{\text {Tip-Alu }}$ is the constriction resistance between the tip and the alumina matrix. Considering the matrix as a semi-infinite medium due to its dimensions in comparison with the thermal exchange radius $r_{\mathrm{ex}}$, the constriction resistance can be expressed as $^{27} R_{\text {Tip-Alu }}=\frac{1}{4 \lambda_{\text {Alu }} r_{\text {ex }}}$ where $\lambda_{\text {Alu }}$ is the thermal conductivity of alumina.

As commented in ref. 20, the intrinsic thermal resistance $R_{\text {Com }}$ does not correspond to the NW intrinsic thermal resistance, but to the local composite (alumina and NW) thermal resistance. Indeed, first, the thermal exchange surface is larger than the NW section; hence the hot tip heats not only the NW but also the surrounding alumina matrix at the same time. In addition, the heat passing through the NW spreads towards the matrix since the NWs are in contact with alumina and NWs and alumina are not expected to have much different thermal conductivities. $R_{\text {Com }}$ can then be expressed as a constriction resistance on a semi-infinite effective medium,

$$
R_{\mathrm{Com}}=\frac{1}{4 \lambda_{\mathrm{Com}} r_{\mathrm{ex}}}
$$

where $\lambda_{\text {Com }}$ is the thermal conductivity of the composite calculated using the effective medium theory: ${ }^{\mathbf{1 4 , 2 0}}$

$$
\lambda_{\mathrm{Com}}=x \lambda_{\mathrm{NW}}+\left(\begin{array}{ll}
1 & x
\end{array}\right) \lambda_{\mathrm{Alu}}
$$

where $x$ is the areal packing density of the NW array and $\lambda_{\mathrm{NW}}$ and $\lambda_{\text {Alu }}$ are the intrinsic NW and the porous alumina matrix thermal conductivities, respectively. In ref. 14, the authors study in detail the validity of two models to describe the thermal exchange between NWs and matrix, namely the effective medium and two-temperature models, when heating a sample made of NWs in a matrix using a modulated heart source. It is demonstrated that when the heat source is modulated at low frequencies $(f<1 \mathrm{MHz})$, which is our case $(f=1 \mathrm{kHz})$, the measured thermal conductivity approaches the thermal conductivity predicted by effective medium theory (eqn (5)) with a thermal conductance of the matrix $/ \mathrm{NW}_{\text {interfaces }}, G_{\text {matrix }} / \mathrm{NW} \rightarrow$ $\infty$. Then, NWs and the matrix are strongly coupled and the heat passing from the tip to the NW spreads to the surrounding matrix. We hence heat the whole composite medium over the thermal penetration length, which is typically several microns at this low frequency.

Then, from the same $\left(V_{3 \omega}\right)_{\text {Tip }}$ image presented in Fig. 1c we measured the $\left(V_{3 \omega}\right)_{\text {Tip }}$ signal on 20 locations on the alumina area for the three samples with porous sizes of $350 \mathrm{~nm}, 220 \mathrm{~nm}$ and $120 \mathrm{~nm}$. The thermal conductivity of the alumina matrix resulted to be $\lambda_{\text {Alu }}=1.38 \mathrm{~W} \mathrm{~K}^{-1} \mathrm{~m}^{-1}$, see Table 1 , for the three templates. The thermal conductivity values are consistent with each other since all the templates were prepared under the same conditions; ${ }^{23,25}$ the only difference is that the pores are widened by chemical etching and the porosity increases. With these values of alumina the mean contact resistances are determined to be $R_{\mathrm{C}}=3.60 \times 10^{6} \mathrm{~K} \mathrm{~W}^{-1}, R_{\mathrm{C}}=3.31 \times 10^{6} \mathrm{~K} \mathrm{~W}^{-1}$ and $R_{\mathrm{C}}=4.12 \times 10^{6} \mathrm{~K} \mathrm{~W}^{-1}$ for alumina with $350 \mathrm{~nm}, 220 \mathrm{~nm}$ and $120 \mathrm{~nm}$ in diameter pores, respectively. Often, the contact resistance is determined by calibration on a material of known thermal conductivity. ${ }^{17,26}$ It is then assumed that $R_{\mathrm{C}}$ does not change from sample to sample and when measuring other materials. Nevertheless, precautions need to be taken since this contact resistance may be highly dependent on various parameters such as the surface roughness or the tip-to-sample contact geometry. In our case, when measuring $\left(R_{\text {eq }}\right)_{\text {Alumina }}$ on the alumina part of the three samples, even if the tip is identical, we measure three different values (Table 1), hence three different contact resistances. We have previously proposed ${ }^{\mathbf{1 9}}$ an original method to determine $R_{\mathrm{C}}$ accurately: from a $3 \omega$-SThM image, we deduce it from the equivalent thermal resistances measured directly on the NWs. This method demands a sample with NWs offering wide diameter dispersion, which is not the case here. But we have also shown ${ }^{19}$ that, determining the mean contact resistance subsequently from the equivalent thermal resistance measured from the same $3 \omega$-SThM image on the matrix of the same sample, the estimated values obtained by both methods differ by less than $1 \%$. Therefore, if it does not seem appropriate to evaluate the contact resistance on a given sample and then use the same value for other samples, measuring $R_{\mathrm{C}}$ on a part of a sample seems to give a reliable value that can be used on another part of the same sample from a thermal image obtained during the same scan under the same experimental conditions, in particular with a contact force between tip and sample maintained constant by the AFM feedback loop.

Afterwards, we take into account a possible $\pm 1 \%$ relative error in the contact resistance. This value, which is also consistent with the standard deviation evaluated on $\left(R_{\text {eq }}\right)_{\text {Alumina }}$ in Table 1 and from which we deduce $R_{\mathrm{C}}$, can appear small in comparison with classical mechanical contact resistance relative variations. Indeed, it only takes into account the repeatability error which is reduced because, from one image, we do 20 measurements on the alumina part, reducing the standard deviation by almost 5 . With this $\pm 1 \%$ possible error, the mean composite intrinsic thermal resistances, $R_{\mathrm{Com}}$, were determined to be $R_{\text {Com }}=(0.760 \pm 0.036) \times 10^{6} \mathrm{~K} \mathrm{~W}^{-1}, R_{\mathrm{Com}}=(1.180 \pm$ $0.033) \times 10^{6} \mathrm{~K} \mathrm{~W}^{-1}$ and $R_{\mathrm{Com}}=(2.36 \pm 0.041) \times 10^{6} \mathrm{~K} \mathrm{~W}^{-1}$ for the P3HT NWs with $350 \mathrm{~nm}, 220 \mathrm{~nm}$ and $120 \mathrm{~nm}$ diameter, respectively. 
From eqn (4) the local thermal conductivity of the composites was deduced to be $\lambda_{\text {Com }}=1.89 \pm 0.08 \mathrm{~W} \mathrm{~m}^{-1} \mathrm{~K}^{-1}, \lambda_{\mathrm{Com}}=$ $1.18 \pm 0.06 \mathrm{~W} \mathrm{~m}^{-1} \mathrm{~K}^{-1}$ and $\lambda_{\mathrm{Com}}=1.31 \pm 0.02 \mathrm{~W} \mathrm{~m}^{-1} \mathrm{~K}^{-1}$, for composites made of P3HT NWs with $350 \mathrm{~nm}, 220 \mathrm{~nm}$ and 120 $\mathrm{nm}$ diameters embedded in the porous alumina matrix, respectively. It is worth mentioning that these values are extremely useful, and relevant, as it constitutes the thermal conductivity values of possible functional devices.

Finally, the intrinsic NW thermal conductivity is calculated using eqn (5). In Table 1 the areal packing density of the NW array evaluated from digital analysis of SEM pictures of the samples' top views, the thermal conductivity of the three different composites (considering it as a mixture of alumina and the P3HT material), and the thermal conductivities of individual P3HT NWs with $350 \mathrm{~nm}, 220 \mathrm{~nm}$ and $120 \mathrm{~nm}$ diameters is shown. The validity of the effective medium theory to determine the thermal conductivity of individual NWs was checked with 3D simulations of the different samples under the same experimental conditions as shown in the ESI, S5. $\dagger$

The results shown in Table 1 clearly evidence the reduction of the thermal conductivity of individual P3HT NWs. For semicrystalline polymers, thermal conductivity is known to depend on both the degree of crystallinity and the orientation of their structural elements, i.e. molecules, aggregates, crystals, etc. $^{28}$ On the one hand, crystals show intrinsically higher conductivity than amorphous regions, in such a way that thermal conductivity of semicrystalline polymers is usually higher than that of amorphous polymers. On the other hand, the orientation phenomenon leads to a large anisotropy in the thermal transport of semicrystalline polymers, which can be commonly understood considering that molecular chains in the crystallites are aligned in a certain direction, thus offering little thermal resistance along that direction. P3HT is known to be a semicrystalline polymer and, thus, the consideration above should be taken into account when studying its thermal transport properties. Recently, Feng et al. have shown that thermal conductivity of P3HT does not depend significantly on density, which can be directly correlated with the degree of crystallinity of the polymer..$^{29}$ They observed an increase of only $12 \%$ of the thermal conductivity between P3HT films having density values around $1 \mathrm{~g} \mathrm{ml}^{-1}$ (which according to Ro et al. corresponds to completely amorphous $\mathrm{P} 3 \mathrm{HT}^{30}$ ) and those having values around $1.6 \mathrm{~g} \mathrm{ml}^{-1}$ (highly crystalline P3HT). This low crystallinity dependence of the thermal conductivity in polymers having medium degrees of crystallinity, like P3HT (the degree of crystallinity of bulk P3HT has been proposed to be somewhat below $50 \%$ (ref. 31)), has been suggested to be a consequence of the difference in elastic properties between amorphous and crystalline regions, which causes a high thermal boundary resistance at the many interfaces between amorphous regions and crystals. $^{28}$

In contrast, orientation phenomena are likely to modify strongly the thermal conductivity of semicrystalline polymers and to induce a large anisotropy as a function of crystallographic directions. Piraux et $a l^{32}$ observed that the thermal conductivity of oriented polyacetylene films (another semicrystalline conjugated polymer) was 15-60 times higher than that of non-oriented polyacetylene. Kilian et $a l .{ }^{33}$ reported that the thermal diffusivity in stretched polyethylene was 50 times higher along the drawing direction than along the perpendicular direction. Moreover, this observation contradicts the behavior of amorphous polyethylene, for which only a 2 fold increase was measured. This fact points out the special relevance of crystal orientation phenomena when dealing with semicrystalline polymers. Likewise, Feng et al. has recently reported strong anisotropic thermal transport in P3HT films along the 3 spatial dimensions. ${ }^{10}$

2D-nanoconfinement, like the one imposed by the cylindrical nanopores of AAO templates, frequently induces a preferential orientation of the confined polymer crystals. ${ }^{34,35}$ Thus, to elucidate whether changes in the orientation of P3HT crystals in the NWs may be at the origin of the reduction of their thermal conductivities, WAXS measurements were carried out for two different spatial directions, i.e. directions parallel and perpendicular to the NW long axis. Note that $2 \mathrm{D}$ patterns were collected in the direction perpendicular to NWs and then converted to one-dimensional scattering profiles by radial averaging along the azimuthal angle. All the samples showed diffraction rings in the perpendicular direction (not shown).

In the experimental geometry in which the wave vector, $Q$, was perpendicular to the NW long axis, the three samples (P3HT NWs of 350, 220 and $120 \mathrm{~nm}$ diameters) showed a diffraction maximum at $2 \theta=5.2^{\circ}$ corresponding to the stacking of the main chain/side-chain layered structure of the P3HT crystal along the $a$ axis $^{36,37}$ (Fig. 2a-c). In general, in the three samples, crystals were preferentially oriented lying with their [100] crystallographic direction perpendicular to the NW long axis and, thus, the [010] direction (the $\pi-\pi$ stacking direction) or the [001] direction lays preferentially parallel to the NW axis (ideally presented in Fig. $2 \mathrm{~d}$ and e). The most plausible orientation is the one in which crystals lay in the $\pi-\pi$ stacking direction parallel to the nanowire long axis, as that orientation is the one fulfilling the Bridgeman mechanism ${ }^{38}{ }^{40}$ for orientations guided by kinetic aspects, as it has been usually observed in commodity polymers confined in nanopores. ${ }^{39,40}$ Such a mechanism dictates that the crystallographic direction with the fastest growth rate aligns parallel to the NW long axis. For the P3HT crystal, the $\pi-\pi$ stacking direction is known to be the fastest growth direction, ${ }^{36,41}$ and thus it is expected to be parallel to the NW long axis. The diffraction peak shows a decrease in intensity and a broadening upon reduction of the diameter of the nanowires from 350 to $120 \mathrm{~nm}$. There are three main reasons for this: (a) the porosity of the alumina template is lower in $120 \mathrm{~nm}$ (8\%) than in $220 \mathrm{~nm}(25 \%)$ than in $350 \mathrm{~nm} \mathrm{(55 \% ),} \mathrm{so} \mathrm{the}$ amount of diffracting P3HT is the lowest in $120 \mathrm{~nm}$. (b) The crystal size is smaller when reducing the wire diameter, so the diffraction peaks become broader and (c) some of the P3HT crystals may tilt under confinement.

The (100) diffraction for $Q$ parallel to the NW long axis was absent in $350 \mathrm{~nm}$ nanowires, while weak (100) peaks become visible for 250 and $120 \mathrm{~nm}$ samples, being more intense in 120 $\mathrm{nm}$ nanowires. This means that as the nanowire diameter is reduced, more and more crystals are tilted toward the [100] direction parallel to the NW axis. Note that in crystals with the 


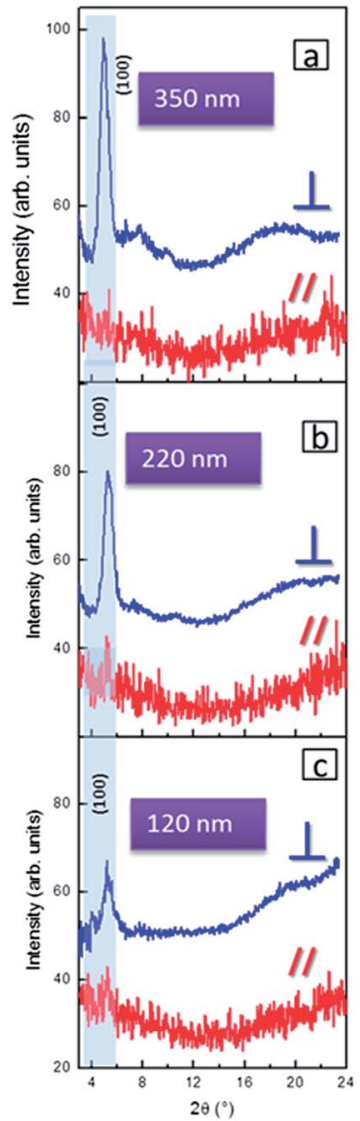

\section{$[100] \perp$ AAO pore}

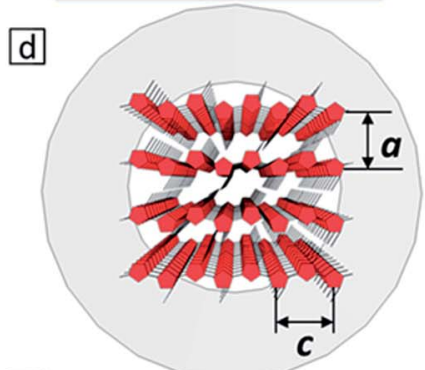

e

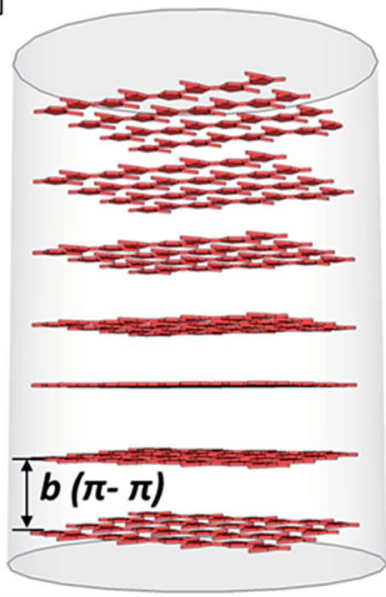

[100] // AAO pore

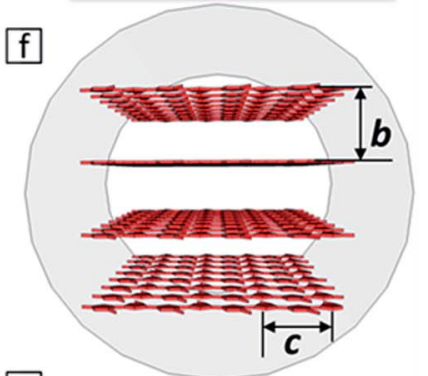

g.

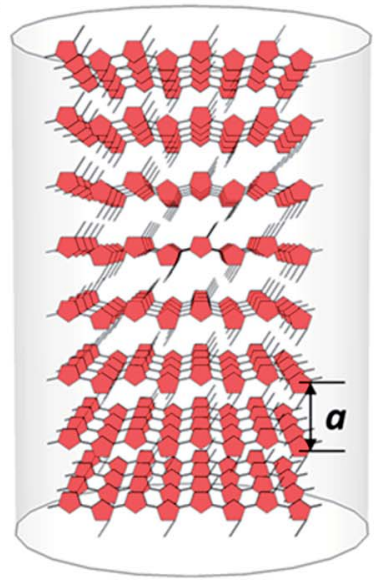

Fig. 2 WAXS diffractograms of ensembles of P3HT NWs in which the wave vector, $Q$, was perpendicular to nanowires (the lower red line with II symbol) and parallel to nanowires (the upper blue line with $\vdash$ symbol) for (a) $350 \mathrm{~nm}$, (b) $250 \mathrm{~nm}$ and (c) $120 \mathrm{~nm}$ NW arrays. Schematic illustrations of the 2 possible ideal spatial orientations of the P3HT crystallite within nanopores from up and transversal perspectives: ( $d$ and e) top and side view of the $b$ axis of the crystal cell ( $\pi \pi$ stacking direction) parallel to the NW long axis which also corresponds to 100 perpendicular, and ( $f$ and g) top and side view of the a axis of the crystal cell ([100] growth direction) parallel to the NW long axis.

[100] direction parallel to the NW axis, the [010] direction is almost perpendicular to the AAO pore walls. Since the [010] direction is that of the fastest growth, P3HT crystallites would tend to grow along that direction, but they impinge on the pore walls and die. This would lead these crystals to be considerably small, which would generate non-well-developed diffraction peaks when measuring in the geometry where $Q$ is parallel to the nanowire axis. This new configuration of the chain is ideally presented in Fig. $2 \mathrm{f}$ and $\mathrm{g}$.

To perform a semiquantitative analysis of the crystal orientation of P3HT NWs, an orientation parameter $\Gamma$, defined as $\Gamma=$ $\gamma_{\vdash} / 1.18 \gamma_{\|}, \gamma_{\vdash}$ and $\gamma_{\|}$being the areas of the (100) peaks in directions perpendicular and parallel to the NW axis, respectively. The coefficient 1.18 was extracted from the ratio $\gamma_{\vdash} / \gamma_{\|}$of the bulk P3HT powder (ESI, Fig. S3†), considering the fact that crystals must be isotropically oriented in that sample and thus $\Gamma$ must be equal to unity (the P3HT was powdered in an agate mortar). In this way, $\Gamma$ is closely related to the preferential orientation of the (100) planes in the NWs. Since $\Gamma>1$ for the three samples, crystals laid with their [100] crystallographic direction preferentially perpendicular to the NW axis and, thus, [010] and/or [001] directions were preferentially parallel to the NW axis (Fig. $2 \mathrm{~d}$ and e). As can be observed in Fig. 3, $\Gamma$ decreased as the pore diameter decreases, suggesting the presence of more and more crystals with [100] parallel to NWs, as ideally presented in Fig. $2 \mathrm{f}$ and g. Note that although we

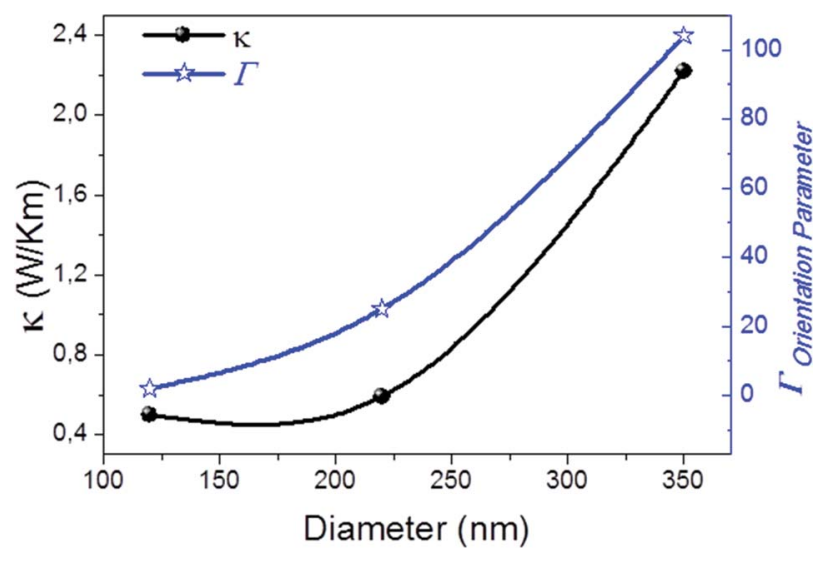

Fig. 3 Plot of the thermal conductivity (black spheres) and the orientation parameter, $\Gamma$, (blue stars) of P3HT NWs as a function of the NW diameter. $\Gamma \quad \gamma_{\vdash} / 1.18 \gamma \|$, where $\gamma_{\vdash}$ and $\gamma \|$ are the areas of the (100) peaks in directions perpendicular and parallel to the NW axis, respectively. The coefficient 1.18 is extracted from the ratio $\gamma_{\vdash} / \gamma_{\|}$of the bulk P3HT. 
cannot assure whether the extended polymer chain direction $(c$ axis) or the $\pi-\pi$ stacking direction ( $b$ axis) is parallel to the NW long axis, both of them are expected to present little thermal resistance in an analogous manner to what occurs with electronic transport (the main electronic conduction in P3HT takes place along the thiophenic backbone and along the $\pi-\pi$ stacking direction). This is because strong conjugated covalent bonds along the chain direction ( [001] direction) and the compact $\pi-\pi$ stacking (along the [010] direction) would facilitate the phonon transport along those crystallographic directions. In contrast, the [100] crystallographic direction is the one along which the alternation of layers of thiophenic chains and aliphatic chains takes place. Thereby, insulating aliphatic regions separate more conductive thiophenic layers, which may introduce additional thermal boundary resistances in the crystal structure along that direction. Furthermore, many authors maintain that medium size alkyl side chains, such as the hexyl groups of P3HT, remain disordered after the crystallization of thiophenic layers, ${ }^{42}$ which would increase further the thermal barriers in those regions. Therefore, we attribute the reduction in thermal conductivity in P3HT NWs, when reducing their diameter, to the decrease of crystals oriented along the [010] direction. These results are qualitatively in accordance with the anisotropy of the thermal conductivity of oriented P3HT films found by Feng et al., ${ }^{10}$ as well as by other authors for non-conjugated polymers confined in nanopores. ${ }^{7}$

Fig. 3 shows that varying the diameter of the nanowire will lead to reduction in its thermal conductivity. These nanowires could be used potentially in different applications in thermal transport engineering because on varying the diameter of a nanowire the changes in its thermal conductivity are appreciable. Therefore, the heat flow across a device could be controlled with a certain magnitude by selecting the appropriate P3HT diameter nanowire.

In summary, this work presents a correlation of the thermal conductivity of 1D semicrystalline polymer nanostructures with the orientation of their crystals. This involves a better understanding of the effects of size confinement in polymers and its correlation with their thermal transport. Particularly, P3HT nanowires of three different diameters were studied and a drastic reduction of their thermal conductivity was observed with decrease in diameter. Such reduction is proposed to be the consequence of an increasing presence of crystals oriented lying with the [100] direction parallel to the nanowire long axis. This analysis evidences the huge potential of nanoscale crystal engineering to modulate thermal transport along the NWs, which may establish the foundations of future nanostructured heat thermal transport engineering for different applications.

\section{Acknowledgements}

Authors thank A. Nogales for WAXS measurements. M. M. R wants to acknowledge JAE-PreDoc for its financial support. ERC 2008 Starting Grant "Nano-TEC" number 240497 and Nanotherm Consolider CSD-2010-00044 projects are acknowledged for financial support.

\section{References}

1 G. M. Whitesides, Small, 2005, 1, 172-179.

2 M. Martín-González, O. Caballero-Calero and P. Diaz-Chao, Renewable Sustainable Energy Rev., 2013, 24, 288-305.

3 M. Muñoz Rojo, O. Caballero Calero, A. F. Lopeandia, J. Rodriguez-Viejo and M. Martin-Gonzalez, Nanoscale, 2013, 5, 11526-11544.

4 H. Ünlü and J. M. N. Horing, Low Dimensional Semiconductor Structures, Springer, Springer Heidelberg, 2013.

5 J. Martín, M. Krutyeva, M. Monkenbusch, A. Arbe, J. Allgaier, A. Radulescu, P. Falus, J. Maiz, C. Mijangos, J. Colmenero and D. Richter, Phys. Rev. Lett., 2010, 104, 197801.

6 S. Shen, A. Henry, J. Tong, R. Zheng and G. Chen, Nat. Nanotechnol., 2010, 5, 251-255.

7 B.-Y. Cao, Y.-W. Li, J. Kong, H. Chen, Y. Xu, K.-L. Yung and A. Cai, Polymer, 2011, 52, 1711-1715.

8 J. Martin, M. Campoy-Quiles, A. Nogales, M. Garriga, M. I. Alonso, A. R. Goni and M. S. Martin-Gonzalez, Soft Matter, 2014, 10, 3335-3346.

9 C. Bounioux, P. Diaz-Chao, M. Campoy-Quiles, M. S. MartinGonzalez, A. R. Goni, R. Yerushalmi-Rozen and C. Muller, Energy Environ. Sci., 2013, 6, 918-925.

10 X. Feng, G. Liu, S. Xu, H. Lin and X. Wang, Polymer, 2013, 54, 1887-1895.

11 J. C. Duda, P. E. Hopkins, Y. Shen and M. C. Gupta, Appl. Phys. Lett., 2013, 102, 251912.

12 L. A. Bottomley, Anal. Chem., 1998, 70, 425R-475R.

13 L. Shi, D. Li, C. Yu, W. Jang, D. Kim, Z. Yao, P. Kim and A. Majumdar, J. Heat Transfer, 2003, 125, 881-888.

14 A. I. Persson, Y. K. Koh, D. G. Cahill, L. Samuelson and H. Linke, Nano Lett., 2009, 9, 4484-4488.

15 K. G. Biswas, T. D. Sands, B. A. Cola and X. Xu, Appl. Phys. Lett., 2009, 94, 223116.

16 T. Borca-Tasciuc, D. A. Borca-Tasciuc and C. Gang, IEEE Trans. Compon. Packag. Technol., 2007, 30, 609.

17 Y. Zhang, C. Hapenciuc, E. Castillo, T. Borca Tasciuc, R. Mehta, C. Karthik and G. Ramanath, Appl. Phys. Lett., 2010, 96, 062107.

18 E. Puyoo, S. Grauby, J.-M. Rampnoux, E. Rouviere and S. Dilhaire, Rev. Sci. Instrum., 2010, 81, 073701-073705.

19 E. Puyoo, S. Grauby, J.-M. Rampnoux, E. Rouviere and S. Dilhaire, J. Appl. Phys., 2011, 109, 024302-024309.

20 M. Muñoz-Rojo, S. Grauby, J. M. Rampnoux, O. CaballeroCalero, M. Martin-Gonzalez and S. Dilhaire, J. Appl. Phys., 2013, 113, 054308.

21 S. Grauby, E. Puyoo, J.-M. Rampnoux, E. Rouvière and S. Dilhaire, J. Phys. Chem. C, 2013, 117, 9025-9034.

22 S. Lefèvre, S. Volz and P.-O. Chapuis, Int. J. Heat Mass Transfer, 2006, 49, 251-258.

23 J. Martín and M. Martín González, Nanoscale, 2012, 4, 5608. 24 J. Martín, C. V. Manzano and M. Martín-González, Microporous Mesoporous Mater., 2012, 151, 311-316.

25 J. Martín, A. Nogales and M. Martín-González, Macromolecules, 2013, 46, 1477-1483.

26 M. Hinz, Appl. Phys. Lett., 2008, 92, 043122-043123. 
27 R. Prasher, Nano Lett., 2005, 5, 2155-2159.

28 C. L. Choy, Polymer, 1977, 18, 984-1004.

29 X. Feng and X. Wang, Thin Solid Films, 2011, 519, 5700-5705. 30 H. W. Ro, B. Akgun, B. T. O'Connor, M. Hammond, R. J. Kline, C. R. Snyder, S. K. Satija, A. L. Ayzner, M. F. Toney, C. L. Soles and D. M. DeLongchamp, Macromolecules, 2012, 45, 6587-6599.

31 Z. Wu, A. Petzold, T. Henze, T. Thurn-Albrecht, R. H. Lohwasser, M. Sommer and M. Thelakkat, Macromolecules, 2010, 43, 4646-4653.

32 L. Piraux, M. Kinany-Alaoui, J. P. Issi, D. Begin and D. Billaud, Solid State Commun., 1989, 70, 427-429.

33 H. G. Kilian and M. Pietralla, Polymer, 1978, 19, 664-672.

34 J. Maiz, J. Martín and C. Mijangos, Langmuir, 2012, 28, 12296-12303.
35 J. Martín, A. Nogales and C. Mijangos, Macromolecules, 2013, 46, 7415-7422.

36 J. A. Lim, F. Liu, S. Ferdous, M. Muthukumar and A. L. Briseno, Mater. Today, 2010, 13, 14-24.

37 T. J. Prosa, M. J. Winokur, J. Moulton, P. Smith and A. J. Heeger, Macromolecules, 1992, 25, 4364-4372.

38 P. W. Bridgman, Proc. Am. Acad. Arts Sci., 1925, 60, 306.

39 J. Martín, J. Maiz, J. Sacristan and C. Mijangos, Polymer, 2012, 53, 1149-1166.

40 M. Steinhart, Adv. Polym. Sci., 2008, 220, 123-187.

41 M. Brinkmann, J. Polym. Sci., Part B: Polym. Phys., 2011, 49, 1218-1233.

42 M. C. Gurau, D. M. Delongchamp, B. M. Vogel, E. K. Lin, D. A. Fischer, S. Sambasivan and L. J. Richter, Langmuir, 2006, 23, 834-842. 